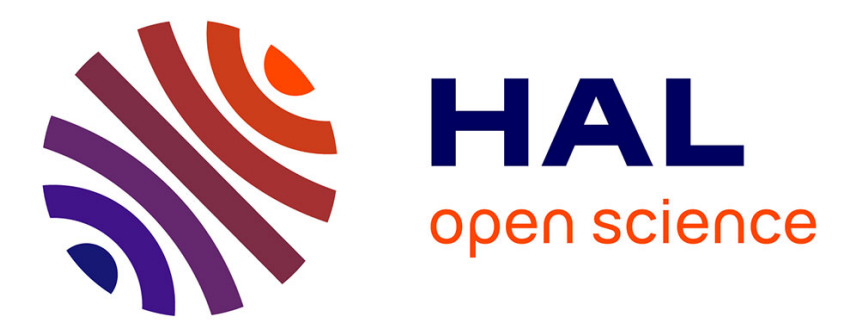

\title{
Circumstellar Disks around young low-mass Stars: observed Properties and Lifetime
}

Anne Dutrey

\section{To cite this version:}

Anne Dutrey. Circumstellar Disks around young low-mass Stars: observed Properties and Lifetime. Comptes Rendus Géoscience, 2007, 339 (14-15), pp.862-871. 10.1016/j.crte.2007.09.010 . hal00255749

\section{HAL Id: hal-00255749 \\ https://hal.science/hal-00255749}

Submitted on 14 Feb 2008

HAL is a multi-disciplinary open access archive for the deposit and dissemination of scientific research documents, whether they are published or not. The documents may come from teaching and research institutions in France or abroad, or from public or private research centers.
L'archive ouverte pluridisciplinaire HAL, est destinée au dépôt et à la diffusion de documents scientifiques de niveau recherche, publiés ou non, émanant des établissements d'enseignement et de recherche français ou étrangers, des laboratoires publics ou privés. 
Les disques circumstellaires entourant les jeunes étoiles de faible masse: propriétés observées et durée de vie

Circumstellar Disks around young low-mass Stars: observed Properties and Lifetime

\author{
A. Dutrey ${ }^{1}$ \\ Laboratoire d'Astrophysique de Bordeaux, OASU, Université de Bordeaux 1, CNRS, 2 rue \\ de l'Observatoire, BP 89, F-33270 Floirac
}

Received

accepted 


\begin{abstract}
Comprendre la formation des systèmes planétaires est l'un des très grands défis de l'astrophysique contemporaine. Jusqu'à il y a environ 15 ans, l'essentiel des connaissances dans ce domaine étaient basés sur la planétologie et l'étude de la formation de notre propre système solaire. Cependant, grace aux progrès en instrumentation astronomique, la nouvelle géneration de telescopes est en train d'ouvrir aux investigations les systèmes planétaires lointains en formation. Les disques proto-planétaires orbitant autour d'étoiles similaires au Soleil jeune sont maintenant facilement observables, aussi bien en optique qu'aux longueurs d'ondes millimétriques. Ces observations commencent à avoir la sensibilité et la résolution angulaire nécessaires pour sonder les régions internes des disques où devraient se former les planètes. Dans cette revue, je présente plusieurs résultats récents qui illustrent ces points et montrent comment les astronomes commencent désormais à contraindre la physique et la chimie de ces disques. Je discute aussi leurs temps caractéristiques et leur evolution.

Je conclus sur quelques questions clefs qui sont parmi les défis de demain si l'on veut comprendre la génèse des planètes.

Unveiling the physical processes leading to planet formation is one of the major challenges of modern astrophysics. Until the last fifteen years, the origin of planetary systems was mostly investigated from observations and modelling of our own Solar System. However, the new generation of astronomical facilities is slowly but surely changing this. Observations of circumstellar disks around young stars similar to the Sun in its infancy are now routinely provided by large astronomical facilities, from the optical wavelengths to the millimeter range. These observations begin to have the sensitivity and angular resolution needed to pro-
\end{abstract}


vide invaluable information inside the regions of disks where planets should form. In this review, I show some new results which illustrate how fast this new domain is evolving and which kind of physical/chemical parameters can be explored. In particular, I present what recent observations tell us about disk lifetime and evolution.

I conclude by some open questions which represent important astrophysical challenges for tomorrow in order to understand how planetary systems form.

Subject headings: star formation - protoplanetary disks - formation of planetary systems - formation stellaire, disques proto-planetaires, formation des systèmes planétaires 


\section{Introduction}

In the early 1980's, optical polarimetric observations [1] revealed the existence of elongated and flattened circumstellar dust material around some Pre-Main-Sequence (PMS) stars such as the low-mass TTauri stars, similar to the Sun when it was about $10^{6}$ years old. A few years later, observations from the InfraRed Astronomical Satellite (IRAS) found significant infrared (IR) excesses around many TTauri stars [2], showing the existence of cold circumstellar dust around these objects. These exciting discoveries motivated several groups to model the Spectral Energy Distribution (SED) of these young stars and to propose an evolutionary scenario [3].

IR excess around TTauri stars was integrated as the signature of a dust disk orbiting the central stars, the material being the residue of the molecular cloud which formed the central star. With total masses (gas + dust) ranging from 0.001 to a $0.1 \mathrm{M}_{\odot}$, most of these disks contain enough gas $\left(\mathrm{H}_{2}\right)$ to allow, in theory, the formation of a giant planet. Hence, they are often called "protoplanetary disks". During this phase, the dust emission is optically thick in the Near-IR (NIR) and the central young star still accretes material from its disk. Such disks are then also called "accretion disks".

Proto-planetary disks orbiting around young stars of a few million years old are now routinely observed by large single-aperture telescopes and interferometers from the radio up to the optical domain. Large optical telescopes provide images of the disk surface where the dust grains scatter the impinging stellar light. When the dust disk is observed close to edge-on, these images reveal that the disks are flaring, as expected from the theory of disks in hydrostatic equilibrium [4]. In the meantime, spectro-imaging of the CO gas provided by millimetre/submillimetre $(\mathrm{mm} / \mathrm{submm})$ arrays have shown that protoplanetary disks are in Keplerian rotation around their central star and that disks can be as large as 500-800 AU, in radius. At the distance of the closest star forming regions ( $\sim 150 \mathrm{pc}$ ), the angular radii 
of these disks are usually within the range $1-3^{\prime \prime}$ or 150-450 AU. However, being mostly heated by the central star, their spectral energy distribution is such that the apparent angular size drops very fast when the observing wavelength goes from the millimeter domain to the optical one. Typically the apparent radius of the thermal emission of a hot dust disk at $10 \mu \mathrm{m}$ would not exceed a few $\mathrm{AU}$ or $\sim 0.1^{\prime \prime}$ while it would be around $1-2^{\prime \prime}$ at $\lambda=1 \mathrm{~mm}$.

Figure 1 is a cartoon showing a "classical" accretion disk orbiting a TTauri star of a few million years and located at the Taurus distance $(150 \mathrm{pc})$. From the observations, one can then naturally distinguish three parts in disks. The very inner disk, located close to its inner radius, is extending up to radius of $\sim 1-3 \mathrm{AU}$ and is only resolved by optical and NIR interferometry. The inner disk is extending up to a radius of $\sim 30 \mathrm{AU}$ and would somewhat correspond in our system to the inner solar system, located up to the first Kuiper Belt objects. The outer disk corresponds to the relatively cold $(\sim 10-50 \mathrm{~K})$ large dust and gas disk usually traced by CO rotation line emission and observed by millimeter and sub-millimeter interferometers such as the IRAM array, the SMA or OVRO.

More generally, these last years have revealed the potential of interferometric techniques as powerful tools to resolve and model such disks. This is true both for small hot inner disks and large colder outer disks. It appears that only aperture synthesis (interferometry) can nowadays technically achieve the angular resolution needed.

\section{Disk Properties: from large to smaller Scales}

The next section presents some major highlights resulting from the technical improvements of large telescopes and interferometers. 
VLTI: Opt./NIR dust + lines VLTI: Mid-IR thermal dust

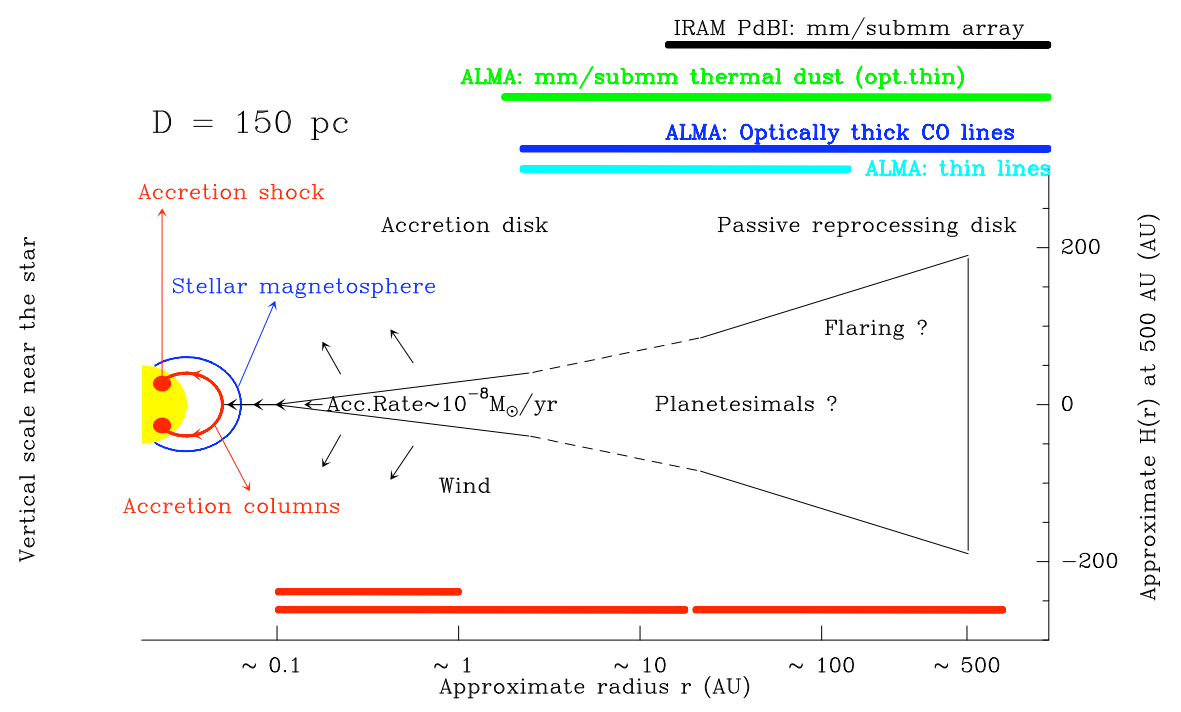

Fig. 1. - Scheme of a disk of a few million years old orbiting a TTauri star located at $150 \mathrm{pc}$ from the Earth. This figure also displays the area sampled in disks by large astronomical facilities depending on their wavelengths, sensitivity and angular resolution. ALMA, the submm array, currently in construction in Chili, will have enough sensitivity and resolution to provide the first images of the inner disk with a linear resolution of a few AUs. Multiwavelengths approaches are necessary to sample the whole disk. Bottom: the three red lines, from left to right, correspond to the very inner disk, the inner disk, the outer disk, respectively. Note that the star is not at the right scale.

Représentation schématique d'un "demi-disque" d'environ $10^{6}$ ans orbitant autour d'une étoile TTauri, à la distance de 150 pc de la Terre. En haut de la figure, sont représentées les zones étudiées avec les grands telescopes sol, en fonction de leur longueur d'onde d'observation, de leur sensibilité et de leur résolution angulaire. L'interféromètre ALMA, en construction au Chili, aura assez de sensibilité et de résolution angulaire pour faire des images des disques internes avec une résolution linéaire de quelques Unités Astronomiques. Ce diagramme montre encore que les analyses multi-longueur d'onde sont nécessaires pour étudier le disque dans son ensemble. Les 3 bandes rouges du bas, de gauche à droite, correspondent à la localisation du disque très interne, du disque interne et du disque externe. L'étoile n'est pas représentée à l'echelle. 


\subsection{Gas Disk observed with millimeter/submillimeter Arrays}

Current $\mathrm{mm} / \mathrm{submm}$ interferometers provide images of the dust and gas outer disks at an angular resolution of $\sim 1^{\prime \prime}$ or $150 \mathrm{AU}$ at the distance of the nearest star forming regions. Molecular hydrogen is, by far, the major constituent of disk (with a dust-to-gas ratio taken as $\sim 100$ ) but remains difficult to observe essentially because it does not possess a dipolar rotational spectrum. Its quadrupolar rotational lines, in the MID-IR domain, mostly trace material at hot/warm temperature in the disk surface close to the star and do not sample the bulk of the cold gas located along the mid-plane. After $\mathrm{H}_{2}$, carbon monoxide is the most abundant molecule (with abundance $\mathrm{CO} / \mathrm{H}_{2}$ of order $\sim 10^{-4}-10^{-6}$ ). Its first rotation lines are observable with current $\mathrm{mm}$ arrays allowing to trace the outer gas disk properties. Since the density in disks is very high $\left(n\left(\mathrm{H}_{2}\right)>10^{6} \mathrm{~cm}^{-3}\right)$, the $\mathrm{J}=1-0$ and $\mathrm{J}=2-1$ CO lines are thermalized by collision with $\mathrm{H}_{2}$ in almost the whole disk. Hence, a simple model of Keplerian disk assuming LTE conditions is sufficient to retrieve the CO disk properties [5].

CO maps not only reveal that disks are in Keplerian rotation around TTauri [6] and Herbig Ae stars [7], they also allow us to retrieve the physical conditions in outer disks. Comparing resolved $\mathrm{CO}$ maps to a disk model by performing a $\chi^{2}$ minimization of the disk parameters gives quantitative information on the density and temperature radial and vertical distributions, provided the analysis properly takes into account the transfer function of the interferometer $[8,9]$.

The radial profiles of the temperature deduced from ${ }^{12} \mathrm{CO}$ images appear to be consistent with models of stellar heating in flared disks, the outer disk being colder than the inner one. Moreover, since the ${ }^{12} \mathrm{CO}$ and ${ }^{13} \mathrm{CO} \mathrm{J}=1-0$ and $\mathrm{J}=2-1$ lines have different opacities, they samples different disk layers and allow us to probe the existence of a vertical temperature gradient. [10] have shown that the "CO disk surface", traced by ${ }^{12} \mathrm{CO}$, is significantly hotter than the mid-plane traced by the optically thinner ${ }^{13} \mathrm{CO} \mathrm{J}=1-0$ 

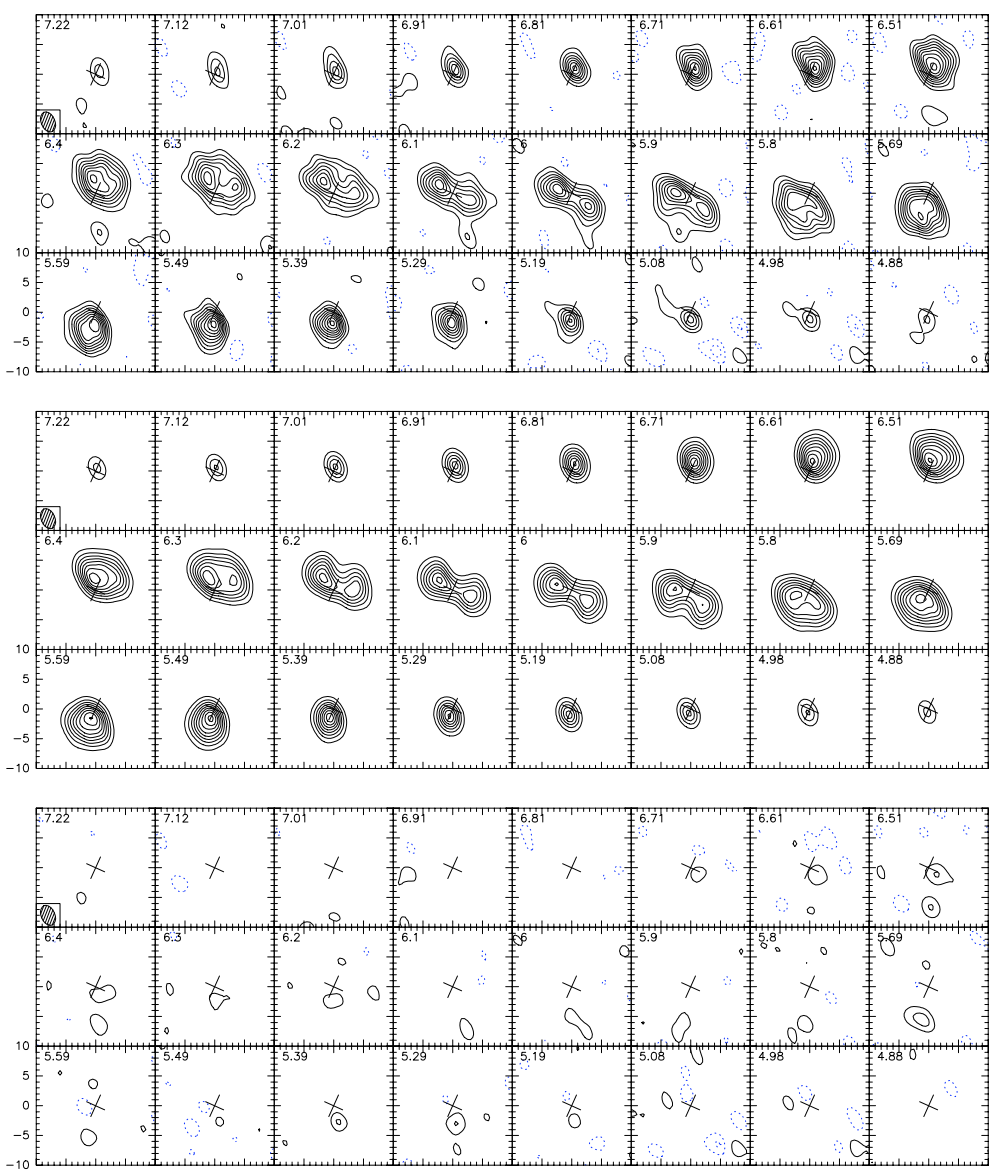

Fig. 2.- From [8]. Observation of the gas disk orbiting the TTauri star DM Tau of $\sim$ $0.5 \mathrm{M}_{\odot}$ and age $\sim 5$ Myrs. CO J=1-0 velocity channel map (spectro-imaging) obtained with the IRAM array at the linear resolution of $\sim 300$ AU. Each box represents a velocity channel. Top: Observed CO J=1-0 channel map. Medium: the best model obtained after $\chi^{2}$ minimizations. Bottom: image of the residuals after subtraction of the best model to the data.

Observation du disque de gaz entourant l'étoile TTauri DM Tau $\left(0.5 \mathrm{M}_{\odot}\right.$, age $\sim 0.510^{6}$ ans). La carte canal à canal de l'émission $\mathrm{CO} J=1-0$ (spectro-imagerie) a été obtenue avec l'interferometre de l'IRAM à la résolution linéaire de $\sim 300$ UA. Chaque boite représente un canal de vitesse. Haut: carte observée. Milieu: meilleur modèle obtenu après minimisation. Bas: image des résidus après avoir fait la différence entre le meilleur modèle et les observations. 
transition, in the DM Tau disk. This effect appears in the region of the disk where the dust is still optically thick in absorption to the stellar radiation while it is already optically thin to its own emission, around $r \sim 50-200$ AU in the DM Tau case. Beyond $r \geq 200 \mathrm{AU}$ where the dust becomes optically thin to both processes, the temperature profile appears vertically isothermal. These observations where the first direct confirmation of models of the vertical thermal structure in disks (e.g. $[11,12,13]$ ). More recently, [14] have extended this type of analysis to several objects, confirming the existence of vertical and radial temperature gradients. They also find that the turbulence appears to be subsonic in the outer part of disks [14].

It is also important to mention that $\mathrm{CO}$ in the gas phase has been observed in all TTauri disks at a temperature which appears to be below the CO freeze out point $(17 \mathrm{~K})$. This suggests that the vertical turbulence should play an important role by providing chemical mixing between the vertical molecular layers.

Searching for other molecules than CO remains sensitivity limited to the most abundant molecules found in molecular clouds. So far, there are only a few attempts to survey a large set of molecules in protoplanetary disks $[15,16,17,9]$. In addition to ${ }^{13} \mathrm{CO}$ and $\mathrm{C}^{18} \mathrm{O}$, only $\mathrm{HCO}^{+}, \mathrm{H}^{13} \mathrm{CO}^{+}, \mathrm{DCO}^{+}, \mathrm{CS}, \mathrm{HCN}, \mathrm{HNC}, \mathrm{DCN}, \mathrm{CN}, \mathrm{H}_{2} \mathrm{CO}, \mathrm{N}_{2} \mathrm{H}^{+}$and $\mathrm{C}_{2} \mathrm{H}$ have been detected. This domain will also benefit from ALMA, the large submm interferometer currently in construction in Chili.

\subsection{MID interferometry: Mineralogy of the Inner Disk}

Continuum observations of the dust, both at mm and NIR wavelengths, imply that the dust in such objects has evolved compared to that found in the interstellar medium. In particular, the particle size has started to increase from sub-micron size up to a few 
centimeters [18]. One key problem in planetary formation is the understanding of the dust evolution in term of grain size, vertical and radial distribution, composition and chemical nature from the inner disk to the outer disk. For example, in the interstellar medium (ISM), the silicate grains are amorphous while in our Solar system, comets and meteorites present crystalline silicates. The new generation of optical, Near-IR and MID-IR interferometers begin to provide direct insights on the inner disks where planetary formation is thought to occur. The observations discussed below present a qualitative but major improvement in the knowledge of the dust properties in inner disks.

[19] recently observed three disks orbiting young stars of $\sim 2 \mathrm{M}_{\odot}$ and a few million year old with MIDI, the $10 \mu \mathrm{m}$ interferometer operated on the Very Large Telescope Interferometer (VLTI) by ESO. They compare the spectra of the silicate bands at $10 \mu \mathrm{m}$ of the very inner disks (radius $\mathrm{R}<2-4 \mathrm{AU}$ ) observed with the VLTI with the spectra of the disks outside $\mathrm{R}>2-4 \mathrm{AU}$. The comparison reveals that in the inner disks the silicate grains appear more crystallized 1) than any other dust observed around young stellar objects and 2) than in the outer part of the disks. Fig.3 shows the spectra. In the very inner disks, the spectra present the three peaks which are characteristics of crystalline silicates, the same peaks are also observed in the spectra of comets such as Halley or Hale-Bopp. The spectra in the outer part of the disks are more similar to those observed in the ISM, characteristics of amorphous silicates. The presence of crystalline silicates in inner disks of a few million years old suggests that silicates crystalize very early in the evolution of the disks, likely before the formation of terrestrial planets.

So far, these recent data bring the first observational constraints on the properties of dust grains at the scale of a few AU in disks. These kind of observations will likely provide in the next years the first detailed analysis of the dust mineralogy in inner disks, leading to a better understanding of the dust evolution in the earliest phases of the planetary 


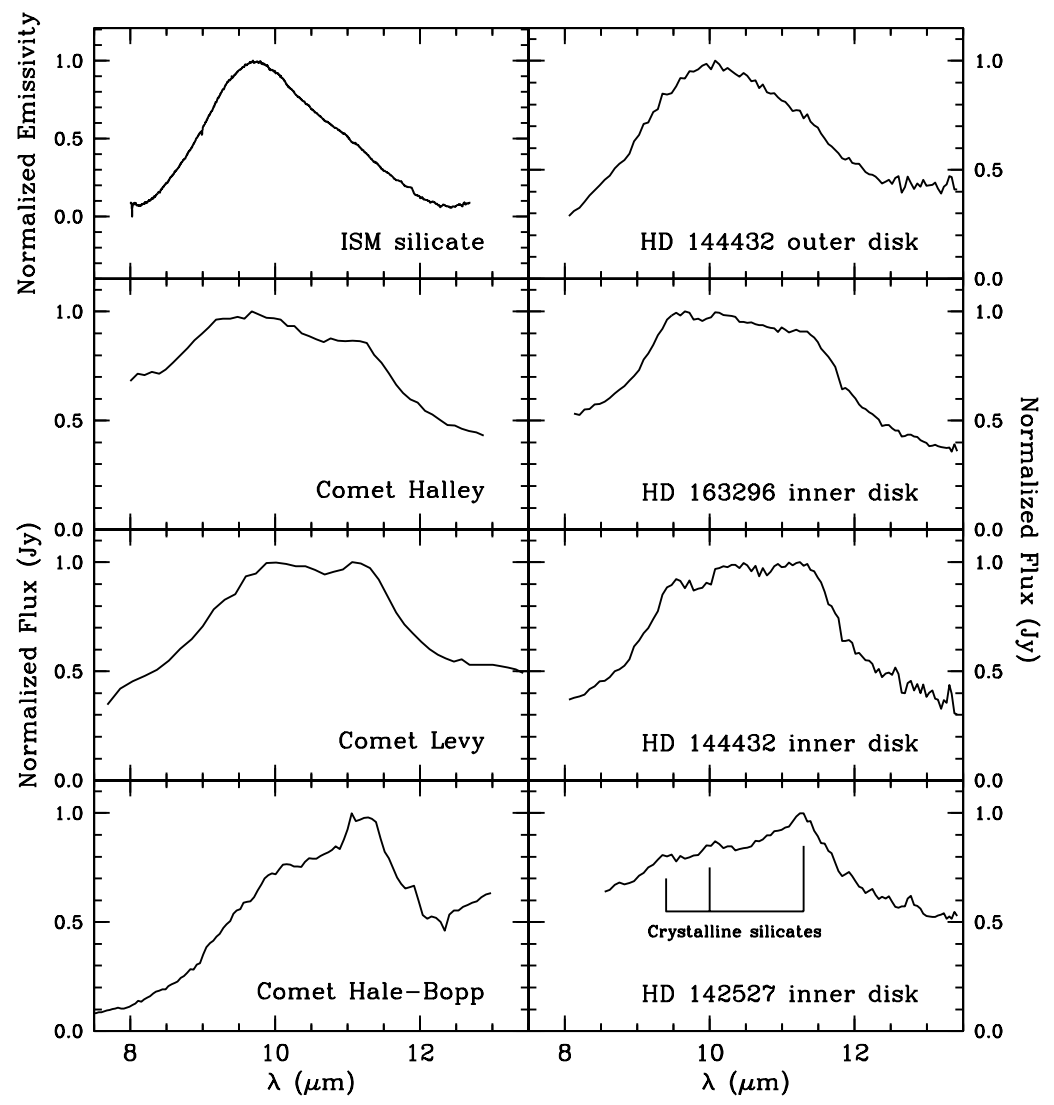

Fig. 3.- From [19]. MIDI Spectra of the Silicate bands around $10 \mu \mathrm{m}$ in disks orbiting three young stars of $\sim 2 \mathrm{M}_{\odot}$ and a few million years old. Top-Left: typical spectrum found in the interstellar medium (ISM), Medium/Bottom-Left: spectra in the comets Halley, Levy and Hale-Bopp Top-Right: spectrum in the outer part (R>2-4 AU) of the disk orbiting HD 144432 (MIDI data), Medium/Bottom-Right: spectra in the inner disks around HD 163296, HD 144432 and HD 142527 (MIDI data). Bottom-Right: the location of the three peaks which are characteristics of crystalline silicates, is given.

Spectres MIDI autour de $10 \mu \mathrm{m}$ (bande des Silicates) de 3 étoiles jeunes d'environ $\sim 2 \mathrm{M}_{\odot}$ et quelques Millions d'années. Haut-Gauche: spectre typique observé dans le milieu interstellaire (ISM). Milieu/Bas-Gauche: spectres observés dans les comètes Halley, Levy et Hale-Bopp,. Haut-Droit: spectre observé dans la partie externe du disque $(\mathrm{R}>2-4 \mathrm{AU})$ de HD 144432 (données MIDI). Milieu/Bas-Droit: spectres observés dans les parties internes des disques autour de HD 163296, HD 144432 et HD 142527 (données MIDI). 
formation.

More generally, recent progresses in observational astrophysics begin to provide the sensitivity and the angular resolution needed to study the regions of proto-planetary disks where planetary formation should occur.

\section{Dissipation of dust and gas Disks}

We have seen in the previous section that the dust found around disks of a few million years has strongly evolved compared to that found in the ISM and in star forming regions. The IR excess is characteristic of the amount of dust found in inner disks, the observation of this excess is often used as a tracer of the inner disk evolution while the submm/mm flux can be reasonably considered as a tracer of the dust in the outer disk. Tracing the evolution of the gas (the bulk of the disk mass) is difficult because it is essentially based on the observation of the low J CO rotational lines. These CO lines also strongly emit in molecular clouds where most of these stars are, at least partly, embedded. As a consequence, the high opacity of the CO lines in the clouds does not allow astronomers to detect CO disks, except if the disks are located at the periphery. It is then difficult to get significant statistics on the gas component evolution. This problem will likely disappear in a few years as soon as the submm array ALMA will provide enough sensitivity to systematically survey the rarer isotopes of $\mathrm{CO}$.

\subsection{The Lifetime of Disks}

Observations of numerous TTauri stars located in star forming regions of various ages has been made by several groups. Figure 4 is a compilation by [20] of the IR excess (traced 
by the H-K excess sampling the very inner disk) versus the age of the stars. There are many uncertainties associated to this kind of analysis, the most important being 1) the errors on the estimate of the IR excess and 2) the derivation of the stellar age (which are taken as the median apparent age of the individual objects belonging to the same cluster). However, there is a clear trend which can be seen on this plot: at age around 1 million years, 80-90 $\%$ of the TTauri stars are surrounded by an innermost dust disk while the fraction of inner disks drops to 30-40\% at 2-3 million years and beyond 5 million years, almost all disks have disappeared.

Comparison of the IR excess with the observations of the dust observed in the $\mathrm{mm} /$ submm domain [21] and CO data [22] suggest that the dissipation of the inner and outer disks are roughly simultaneous. However, since the statistics in the $\mathrm{mm} / \mathrm{submm}$ domain are poor, it is difficult to derive quantitative numbers, yet.

Nevertheless, taking into account the various types of surveys and their results, it seems reasonable to conclude that the dissipation is globally a fast process since more than half of the inner disks disappear after 2-3 million years.

\subsection{Dissipation of Disks}

There are many physical reasons which should explain the apparent dispersal of disks:

- The formation of a planetary system

- The formation of a binary or a multiple stellar system

- The tidal truncation by stellar encounters

- The photo-evaporation of the disk 


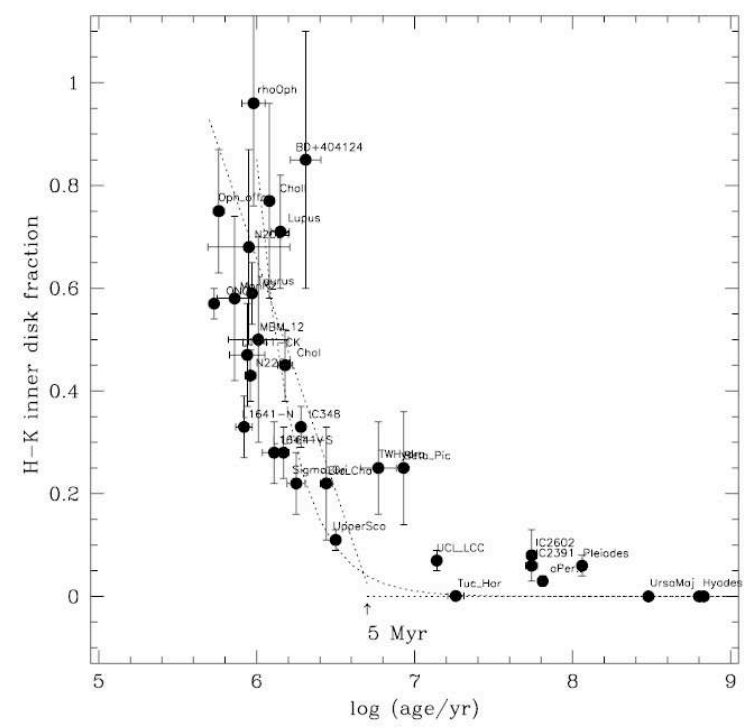

Fig. 4.- [20]. Observations of the IR excess in disks, traced by the H-K excess, the difference of magnitudes $\mathrm{H}(1.6 \mu \mathrm{m})$ and $\mathrm{K}(2.2 \mu \mathrm{m})$. It samples the innermost disk $\sim 0.1-1 \mathrm{AU}$. The diagram has been obtained by compiling the observations of $\sim 3000$ stars located around the Sun $(\sim 50-500 \mathrm{pc})$ and their stellar properties (spectral type, optical luminosity, IR photometry).

Observation de l'excès IR dans les disques, caractérisé par H-K, la différence de magnitude entre la bande $\mathrm{H}(1.6 \mu \mathrm{m})$ et la bande $\mathrm{K}(2.2 \mu \mathrm{m})$. H-K trace la poussière dans la partie très interne du disque $\sim 0.1-1$ AU. Ce diagramme a été obtenu après compilation des observations de 3000 étoiles réparties autour du Soleil à $\sim 50-500$ pc et de leurs propriétés stellaires (type spectral, luminosité, photométrie IR). 
Large optical/NIR surveys of star forming clusters have shown that many stars form in binary and/or multiple systems (eg [23]). The formation of a binary or a multiple stellar system strongly affects the original distribution of material which is tidally disrupted. For example, observations of the TTauri binary system GG Tau [5] have shown that the gas and the dust are located inside a circumbinary disk orbiting the binary while very small individual disks exist around each stellar components, inside the Roche lobe. Hence, even if the innermost disks can exist, statistics provided by studies as those presented above may be partially biased by the presence in the samples of unresolved multiple stellar systems which are not yet known.

In some clusters, such as $\rho$ Ophiuchus or Orion, the stellar density can be as high as $2-5 \times 10^{4} \mathrm{pc}^{-3}$ (eg [24] and external tidal truncations of disks by stellar encounters should be a relatively common phenomenon. For a matter of sensitivity and angular resolution, we do not have yet enough resolved images of disks in these clusters to estimate the importance of this effect. It is however reasonable to take it seriously into account, at least as a phenomenon which should be responsible for the existence of smaller disks in dense stellar clusters.

The photo-evaporation of disks is invoked as a possible phenomenon responsible for the disruption of inner disks [25]. UV photons coming from the young stars reach the disk surface where they can create a ionization front, heating the material behind. The ionized gas becomes gravitationally unbound and flows in a wind away from the disk surface. This phenomenon is observed for the proplyds orbiting TTauri stars located in the Orion Trapezium region [26], close to the OB association. The strong ionizing UV flux from the OB stars photo-evaporates the outer disks in their surroundings. Alexander and collaborators have shown that in some cases photo-evaporation should clear the inner disk very efficiently, creating a central hole, and disrupt the whole disk on a time-scale of about 
$5 \cdot 10^{5}$ years after a disk lifetime of 2-3 million years. This physical process has then to be taken into account because the time-scales (both lifetime and dispersal) are those which are observed for TTauri disks, as illustrated in the Section 3.1. However, there is yet no clear observational evidences of the importance of this process for the disk clearing. Dedicated observations, such as sensitive and resolved $\mathrm{mm} / \mathrm{submm}$ images of inner disks, have to be performed in the next years in order to disentangle between the various scenarii which can be responsible for the existence of an inner hole in disks: photo-evaporation or planetary formation.

Moreover, whatever are the frequencies of the various physical processes described in this section, all of them may affect planet formation since they have a direct impact on the dust and gas distribution close to the stars with the same time-scales.

\section{Towards Planetary Formation: the LkCa15 case}

The extension of the IRAM array baselines performed in summer 2006 provides an angular resolution of 0.3 " which corresponds to $\sim 40$ AU at the Taurus distance; such a linear resolution is of order of our Solar System size. Using this new configuration of the IRAM array, [27] have observed the disks surrounding MWC480 and LkCa15, two young stars of a few million years old and masses of $\sim 2$ and $1 \mathrm{M}_{\odot}$, respectively. Fig.5 presents the images of the thermal emission of the dust at 2.8 and $1.3 \mathrm{~mm}$ around MWC480 and LkCa15. The emission from the dust disk of MWC480 is strongly centrally peaked, as expected from a standard density distribution while the central part of the LkCa15 disk appears partly devoid of dust. The best modelling of the data confirm the visual impression in both cases. The dust disk of LkCa15 exhibits a central cavity of radius $46 \pm 3$ AU. Such a cavity is not detected at NIR wavelengths because, contrary to the $\mathrm{mm} / \mathrm{submm}$ range, the high opacity of the dust in the NIR precludes the detection of cavities corresponding to 
low or moderate density contrast $(\sim 10-100)$. The most probable explanation for such a hole is the presence of a massive planet of 5-10 masses of Jupiter orbiting at $\sim 30$ AU from the star or a low-mass companion $\left(<0.2 \mathrm{M}_{\odot}\right)$.

The LkCa15 disk has been observed at mm wavelengths with lower angular resolutions (ranging from to 200 to $70 \mathrm{AU}$ ) by several interferometers. None of these observations were suggesting such a wide cavity. This example illustrates how an increase in linear resolution (and sensitivity) can change the physics of an astrophysical object, as soon as the spatial scales needed to study the physical processes are reached.

\section{Summary and Open Questions}

In the last fifteen years, resolved observations, from the $\mathrm{mm}$ to the optical domain, of the circumstellar disks orbiting TTauri stars have opened the processes leading to the formation of planetary systems to direct investigations. Nowadays, we are far from understanding the whole process but the characterization of the physical and chemical properties of these proto-planetary disks represents a major step since planets are built from the disk material. Our current knowledge allows us to tell that:

- Many disks can have large outer radii, within the range $100-800$ AU.

- Disks are usually in Keplerian rotation.

- Optical images reveal that disks are flaring.

- Vertical temperature gradients in proto-planetary disks appear to be compatible with 

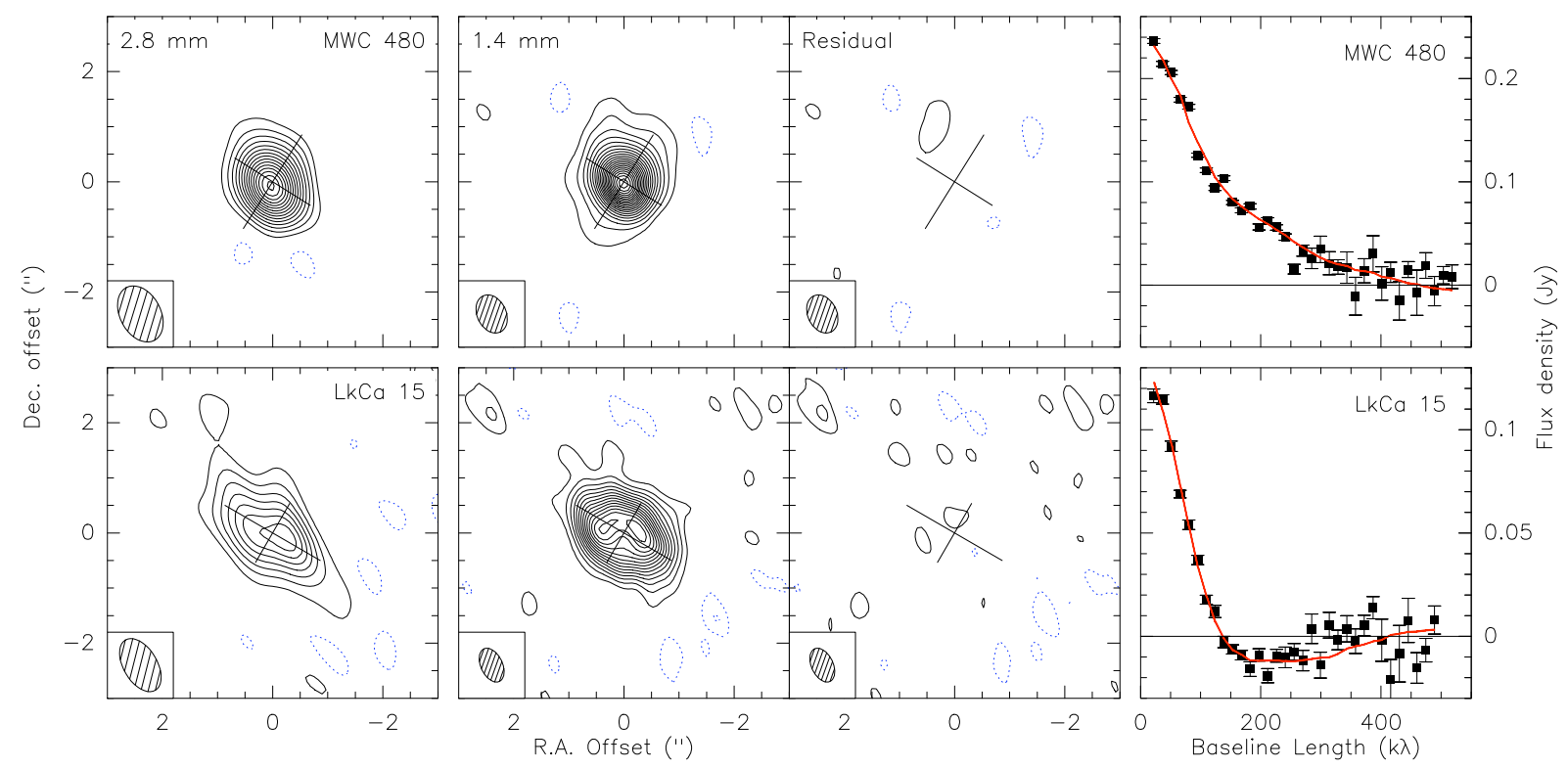

Fig. 5.- From [27]. IRAM interferometer observations of the dust disks surrounding MWC480 and LkCa15 at 1.3 and $2.8 \mathrm{~mm}$. From Left to Right: $2.8 \mathrm{~mm}$ images, $1.3 \mathrm{~mm}$ images, residual images after subtracting the best model to the data, circularly averaged visibility versus baselines length (filled squares) and best model (black curve). Top: MWC480. Bottom: LkCa15. MWC480 and LkCa15 are young stars of a few million years and masses of 2 and $1 \mathrm{M}_{\odot}$, respectively. Contrary to previous observations at low angular resolution, these observations performed with a linear resolution of $\sim 40 \mathrm{AU}$ at $\lambda=1.3 \mathrm{~mm}$ reveal, for the first time, that the inner dust disk of LkCa15 is partly devoid of dust.

Observations IRAM de l'émission thermique des disques de poussières entourant MWC480 et LkCa15. De gauche à droite: images obtenues à $2.8 \mathrm{~mm}$, à $1.3 \mathrm{~mm}$, image des résidus après soustraction du meilleur modèle aux données, visibilites moyennées en fonction de la ligne de base (carrés pleins) et meilleur modèle (courbe noire). Haut: pour MWC480. Bas: pour LkCa15. 
heating by the central star, in agreement with disk models.

- Both mm and optical data show that grains in disks are more evolved than in the parent molecular clouds, with sizes up to a few centimeters.

- NIR surveys suggest that inner disk typical lifetimes are 2-3 million years, beyond 5 million years, most proto-planetary disks may have disappeared.

We have seen through several examples that multi-wavelength approaches are required to get a global understanding of the physical properties of these disks. Optical to MID-IR interferometry has started to unveil the physics of the inner disk. The inner disk will be imaged by the next generation of instruments. With its capability of reaching AU-scale angular resolution at wavelengths where dust has a moderate opacity, ALMA is expected to be the premium instrument to probe these regions and allow astronomers to constrain the models of planet formation. ALMA will also have the potential of providing much higher angular resolution images of the emission of molecular lines allowing detailed modelling of the gas content and of the disk kinematics.

The forthcoming generation of astronomical facilities will likely change our views on planetary formation. In particular, they will bring new information on the following fundamental questions:

- What is the impact of the parent cloud and its vicinity on the disk properties and its evolution?

- What is the feedback of the young star on the gas and dust disk and its evolution (photo-evaporation - photo-chemistry at the disk surface) and how does it affect planetary formation? 
- What are the time-scales for grain growth and for vertical settlement of the dust on the disk mid-plane?

- More generally, what is the mass distribution (gas + dust) radially and vertically in proto-planetary disks and how does it evolve with time?

- What are the indirect/direct evidences of the formation of a planet in a proto-planetary disk ?

Finally, the comparison of these proto-planetary disks which are expected to be representative of the first phases of a planetary system with our own Solar system remains fundamental, as a guideline for each other domain. In the forthcoming years, the understanding of the dynamical and physical/chemical evolution of the dust and gas content in disks will naturally provide a better estimate of the chronology of the planetary formation and a better understanding of the formation of our own Solar System.

Anne Dutrey thanks her collaborators: S.Guilloteau, M.Simon, E.Dartois and V.Piétu for a fruitful long term collaboration. Drs L.Hillenbrand and R.Van Boekel are acknowledged for providing figures.

\section{References}

[1] H. Elsasser and H. J. Staude. On the Polarization of Young Stellar Objects. A\&A, 70:L3+, October 1978.

[2] S. M. Rucinski. IRAS observations of T Tauri and post-T Tauri stars. AJ, 90:2321-2330, November 1985.

[3] F. C. Adams, C. J. Lada, and F. H. Shu. Spectral evolution of young stellar objects. ApJ, 312:788-806, January 1987. 
[4] J. E. Pringle. Accretion discs in astrophysics. ARA\&A, 19:137-162, 1981.

[5] A. Dutrey, S. Guilloteau, and M. Simon. Images of the GG Tauri rotating ring. A\&A, 286:149-159, June 1994.

[6] D. W. Koerner, A. I. Sargent, and S. V. W. Beckwith. A rotating gaseous disk around the T Tauri star GM Aurigae. Icarus, 106:2-+, November 1993.

[7] V. Piétu, A. Dutrey, and C. Kahane. A Keplerian disk around the Herbig Ae star HD 34282. A\&A, 398:565-569, February 2003.

[8] S. Guilloteau and A. Dutrey. Physical parameters of the Keplerian protoplanetary disk of DM Tauri. A\&A, 339:467-476, November 1998.

[9] A. Dutrey, S. Guilloteau, and P. Ho. Interferometric spectro-imaging of molecular gas in proto-planetary disks. In Protostars and Planets V, 2007. http://www.ifa.hawaii.edu/UHNAI/ppv.htm.

[10] E. Dartois, A. Dutrey, and S. Guilloteau. Structure of the DM Tau Outer Disk: Probing the vertical kinetic temperature gradient. A\&A, 399:773-787, February 2003.

[11] E. I. Chiang and P. Goldreich. Spectral Energy Distributions of T Tauri Stars with Passive Circumstellar Disks. ApJ, 490:368-+, November 1997.

[12] P. D'Alessio, J. Canto, N. Calvet, and S. Lizano. Accretion Disks around Young Objects. I. The Detailed Vertical Structure. ApJ, 500:411-+, June 1998.

[13] P. D’Alessio, N. Calvet, L. Hartmann, S. Lizano, and J. Cantó. Accretion Disks around Young Objects. II. Tests of Well-mixed Models with ISM Dust. ApJ, 527:893-909, December 1999.

[14] V. Piétu, A. Dutrey, and S. Guilloteau. Probing the structure of protoplanetary disks: a comparative study of DM Tau, LkCa 15, and MWC 480. A\&A, 467:163-178, May 2007. 
[15] A. Dutrey, S. Guilloteau, and M. Guelin. Chemistry of protosolar-like nebulae: The molecular content of the DM Tau and GG Tau disks. A\&A, 317:L55-L58, January 1997.

[16] J. H. Kastner, B. Zuckerman, D. A. Weintraub, and T. Forveille. X-ray and molecular emission from the nearest region of recent star formation. Science, 277:67-71, 1997.

[17] G.-J. van Zadelhoff, E. F. van Dishoeck, W.-F. Thi, and G. A. Blake. Submillimeter lines from circumstellar disks around pre-main sequence stars. A\&A, 377:566-580, October 2001.

[18] L. Testi, A. Natta, D. S. Shepherd, and D. J. Wilner. Large grains in the disk of CQ Tau. A\&A, 403:323-328, May 2003.

[19] R. van Boekel, M. Min, C. Leinert, L. B. F. M. Waters, A. Richichi, O. Chesneau, C. Dominik, W. Jaffe, A. Dutrey, U. Graser, T. Henning, J. de Jong, R. Köhler, A. de Koter, B. Lopez, F. Malbet, S. Morel, F. Paresce, G. Perrin, T. Preibisch, F. Przygodda, M. Schöller, and M. Wittkowski. The building blocks of planets within the 'terrestrial' region of protoplanetary disks. Nature, 432:479-482, November 2004.

[20] Hillenbrand L. Observational constraints on dust disk lifetime: Implications for planet formation. In A Decade of Discovery: Planets Around Other Stars, 2006.

[21] S. M. Andrews and J. P. Williams. Circumstellar Dust Disks in Taurus-Auriga: The Submillimeter Perspective. ApJ, 631:1134-1160, October 2005.

[22] G. Duvert, S. Guilloteau, F. Ménard, M. Simon, and A. Dutrey. A search for extended disks around weak-lined T Tauri stars. A\&A, 355:165-170, March 2000.

[23] R. Köhler, M. G. Petr-Gotzens, M. J. McCaughrean, J. Bouvier, G. Duchêne, A. Quirrenbach, and H. Zinnecker. Binary stars in the Orion Nebula Cluster. A\&A, 458:461-476, November 2006. 
[24] L. A. Hillenbrand and L. W. Hartmann. A Preliminary Study of the Orion Nebula Cluster Structure and Dynamics. ApJ, 492:540-+, January 1998.

[25] R. D. Alexander, C. J. Clarke, and J. E. Pringle. Photoevaporation of protoplanetary discs - II. Evolutionary models and observable properties. MNRAS, 369:229-239, June 2006 .

[26] J. Bally, R. S. Sutherland, D. Devine, and D. Johnstone. Externally Illuminated Young Stellar Environments in the Orion Nebula: Hubble Space Telescope Planetary Camera and Ultraviolet Observations. AJ, 116:293-321, July 1998.

[27] V. Piétu, A. Dutrey, S. Guilloteau, E. Chapillon, and J. Pety. Resolving the inner dust disks surrounding LkCa 15 and MWC 480 at mm wavelengths. A\&A, 460:L43-L47, December 2006. 